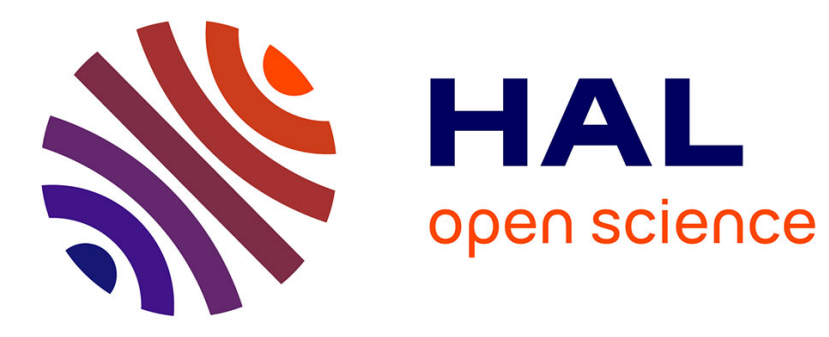

\title{
Adaptive matched filters for contrast imaging
}

Sébastien Ménigot, Iulian Voicu, Anthony Novell, Mélouka Elkateb Hachemi

\author{
Amar, Jean-Marc Girault
}

\section{To cite this version:}

Sébastien Ménigot, Iulian Voicu, Anthony Novell, Mélouka Elkateb Hachemi Amar, Jean-Marc Girault. Adaptive matched filters for contrast imaging. Ultrasonics Symposium (IUS), 2010 IEEE International, Oct 2010, San Diego, United States. pp.1728 - 1731, 10.1109/ULTSYM.2010.5935573 . hal-00626940

\section{HAL Id: hal-00626940 https://hal.science/hal-00626940}

Submitted on 27 Sep 2011

HAL is a multi-disciplinary open access archive for the deposit and dissemination of scientific research documents, whether they are published or not. The documents may come from teaching and research institutions in France or abroad, or from public or private research centers.
L'archive ouverte pluridisciplinaire HAL, est destinée au dépôt et à la diffusion de documents scientifiques de niveau recherche, publiés ou non, émanant des établissements d'enseignement et de recherche français ou étrangers, des laboratoires publics ou privés. 


\title{
Adaptive matched filters for Contrast Imaging
}

\author{
Sébastien Ménigot ${ }^{1}$, Iulian Voicu ${ }^{1}$, Anthony Novell ${ }^{1}$, Melouka Elkateb Hachemi Amar ${ }^{1}$ and Jean-Marc \\ Girault $^{1}$, Member, IEEE \\ 1 Université François Rabelais de Tours, Tours, France \\ INSERM, U930, Tours, France \\ CNRS, ERL 3106, Tours, France
}

\begin{abstract}
The ultrasound (US) contrast imaging is a promising technique. Currently the scientific community of this field seeks US excitations which should make possible the optimization of the acoustic contrast. Two matched filters (MF) techniques are used to improve the image contrast. The first technique is an adaptive MF technique and the second is a RLS technique derived from identification theory.
\end{abstract}

The system proposed is a close loop system which optimizes the power backscattered by microbubbles. After having transmit a first signal, the backscattered power is optimized by transmitting the matched filtered signal at each iteration. This process is iterated until convergence.

Simulations are carried out for encapsulated microbubbles of 2 microns by considering the modified Rayleigh-Plesset equation for a $2.25 \mathrm{MHz}$ transmitted frequency and for various pressure levels $(20 \mathrm{kPa}$ up to $420 \mathrm{kPa})$. In vitro, experiments are carried out by using two transducers a transducers which were placed perpendicularly. The signal was transmitted through a $2.25 \mathrm{MHz}$ transducer. Responses of a 1/2000 diluted solution of SonoVue ${ }^{\mathrm{TM}}$ were measured by a 3.5 $\mathrm{MHz}$ transducer. Each experiment has been realized with three pressure levels $(127,244$ and $370 \mathrm{kPa})$.

We show through simulations and through in vitro experiments that our adaptive imaging technique gives in case of simulations a gain which can reach $12 \mathrm{~dB}$ compared to the traditional technique and for in vitro experiments, the MF gives a gain which can reach $4.5 \mathrm{~dB}$ whereas the MF derived from identification theory can reach $6 \mathrm{~dB}$.

Keywords - Matched filter, adaptive, contrast imaging, identification system.

\section{INTRODUCTION}

Conventional ultrasound contrast imaging systems use a fixed transmit frequency. However it is known that the insonified medium (microbubbles) is time-varying and therefore an adapted time-varying excitation is expected. Contrary to the work presented in [1] which optimized the transmit frequency of a fixed shape, we suggest here an adaptive imaging technique which chooses the wave shape

E-mail jean-marc.girault@univ-tours.fr

2010 IEEE. Reprinted, with permission, from Sébastien Ménigot, Iulian Voicu, Anthony Novell, Melouka Elkateb Hachemi Amar and Jean-Marc Girault,Adaptive matched filters for contrast imaging, 2010 IEEE International Ultrasonics Symposium (IUS), 11-14 Oct. 2010. This material is posted here with permission of the IEEE. Such permission of the IEEE does not in any way imply IEEE endorsement of any of the Université François Rabelais de Tours' products or services. Internal or personal use of this material is permitted. However, permission to reprint/republish this material for advertising or promotional purposes or for creating new collective works for resale or redistribution must be obtained from the IEEE by writing to pubspermissions@ieee.org.

DOI : 10.1109/ULTSYM.2010.5935573 that maximizes the contrast tissue ratio (CTR).

$$
C T R=\frac{E_{\text {bubbles }}}{E_{\text {tissue }}}
$$

with $E_{\text {bubbles }}$ the microbubbles backscattered power and $E_{\text {tissue }}$ the tissue backscattered power. This ratio (eq 1) can be maximized if the backscattered power of the microbubbles is maximized.

We tackled the problem by proposing an adaptive imaging technique derived from the identification theory [2]. The use of an adaptive technique is justified by the fact that:

1. during the clinical examination, the insonified medium perfused by the microbubbles is a non-stationary medium (the concentration and the size of microbubbles change...)

2. the pressure level is unknown because of the diffraction and attenuation effects which vary from one patient to another ;

3 . the size and the distribution of the UCA are not precisely known and can differ from one sample to another.

Two matched filters (MF) techniques were used to improve the image contrast. The first technique was an adaptive MF technique and the second was a Recursive Least Squares (RLS) technique.

\section{Materials AND Methods}

\section{A. Experimental Setup}

Each method was compiled using Matlabß (Mathworks, Natick, MA). The resulting excitation signal was transmitted through a GPIB port (National Instruments, Austin, TX) to an arbitrary function generator (33220A, Agilent, Palo Alto, CA). The signal was then amplified using a power amplifier (Amplifier Research 150A100B, Souderton, PA) and transmitted to a $2.25 \mathrm{MHz}$ PZT single element transducer (Sofranel, Sartrouville, France) focused at 55 $\mathrm{mm}$ and with a fractional bandwidth of $74 \%$ (fig. 1).

The responses of a $1 / 2000$ diluted solution of SonoVue ${ }^{\mathrm{TM}}$ microbubbles (Bracco Research, Geneva, Switzerland) were measured by a $3.5 \mathrm{MHz}$ PZT single element transducer with a fractional bandwidth of $63 \%$, placed perpendicularly to the transmitter, also focused at $55 \mathrm{~mm}$ and used in receiver mode.

The echoes measured were amplified by $30 \mathrm{~dB}$ (Panametrics, Sofranel, Sartrouville, France) and then visualized on a digital oscilloscope (Tektronix, Beaverton, OR). Signals 


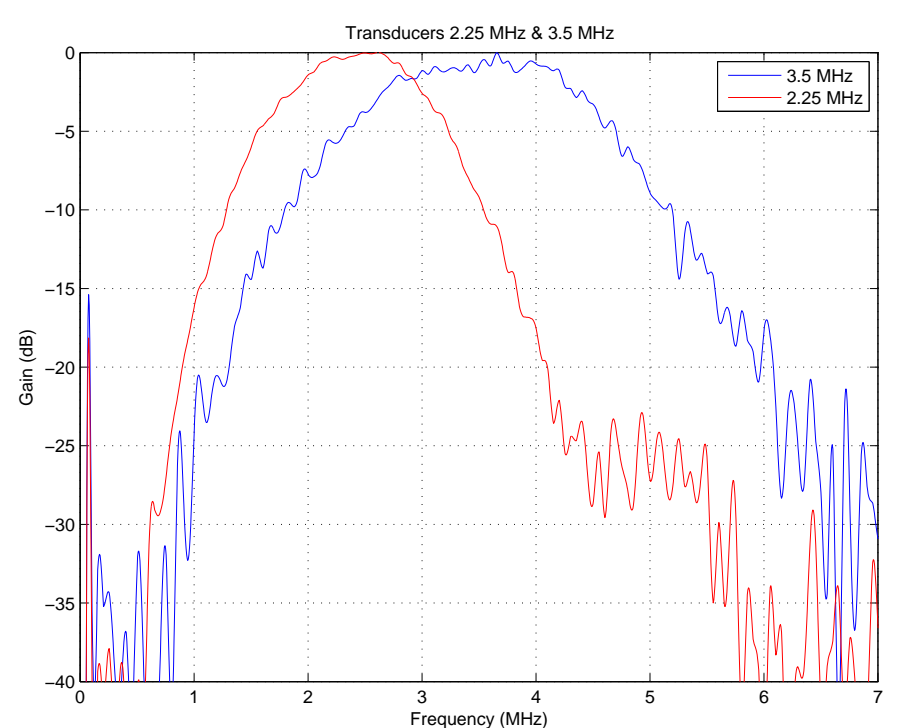

Fig. 1

BANDWIDTH OF TRANSDUCERS

were transferred to a personal computer through a GPIB port for further analysis.

\section{B. Explored Medium}

The ultrasound contrast agent composed of Sonovue ${ }^{\mathrm{TM}}$ microbubbles. The microbubble gas was composed of sulfur hexafluoride $\left(\mathrm{SF}_{6}\right)$ and the bubble shell was a phospholipid monolayer 3]. The mean diameter was $4.5 \mu \mathrm{m}$ [4] and the shell thickness $\left(d_{S e}\right)$ was $1 \mathrm{~nm}$ [5]. The mechanical shell properties were the shear modulus $G_{s} 46 \mathrm{MPa}$ [6] and the shear viscosity $1 \mathrm{~Pa} \cdot \mathrm{s}$.

Numerical simulations for encapsulated contrast microbubbles were solved using Bubblesim [7] from the modified Rayleigh-Plesset equation (eq. 2):

$\ddot{R}(t) \dot{R}(t)+\frac{3}{2} \dot{R}(t)^{2}+\frac{p_{0}+p_{i}(t)-p_{L}(t)}{\rho_{L}}-\frac{R(t)}{\rho_{L} c_{L}} \dot{p}_{L}(t)=0$,

with $R$ the instantaneous radius of the microbubble and their derivatives, $p_{0}$ the static pressure, $p_{i}$ the instantaneous acoustic pressure, $p_{L}$ the liquid pressure on the surface of the microbubble, $\rho_{L}$ the liquid density and $c_{L}$ the velocity of sound in the liquid. The Sonovue ${ }^{\mathrm{TM}}$ properties were used for the microbubble parameters.

The transducer effects were considered: each transmitted and received signal was filtered by the bandwidth of the transmitter and the receiver transducers, respectively.

\section{Methods}

Improve the CTR consistes in maximizing the power backscattered by the microbubble. This maximisation problem can be seem as an optimal command issue for which the best excitation is seeking. In optimal command and for linear system, the best excitation is obtained by time reverse the backscattered signal of the microbubble. We propose two techniques : a standard time reversal technique and a filtered time reversal technique.

\section{C.1 Matched Filter - Time Reversal Technique}

This technique is described in the figure 2 and consisted in :

1. sending throught the transducer and the medium a sinus wave train;

2. measuring the backscatterd signal of the microbubble;

3. time-reversing the signal;

4. normalizing the excitation to ensure the same power in the first step.

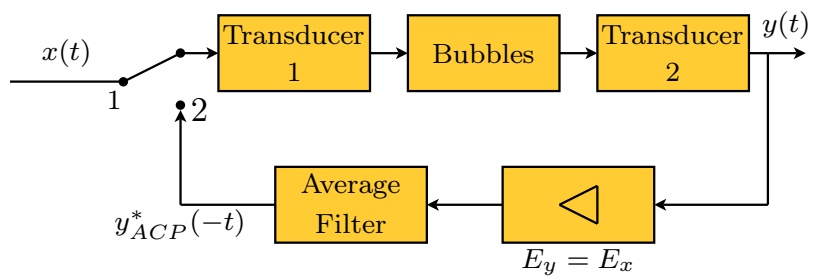

Fig. 2

SCheme of the Bubble System ASsociated With the Matched FILTER

\section{C.2 Recursive Least Squares Filter}

The signal $x(t)$ was injected into the bubble system and into the autoregressive model. The switch was in position 1 in figure 3 . The discretized signal $y(n)$ was modelized by a fourth-order RLS filter (AR-4). This filter minimized the error $e(n)$ between the input and output such as:

$$
e_{n}=y_{n}-x_{n}^{T} \theta_{n-1},
$$

where $x$ was the input of the bubble system, $y_{n}$ the filtered signal at the time $n$ and $\theta$ the vector of filter coefficients. We therefore obtained the filter coefficients $\theta$ [8] by the following equation:

$$
\theta_{n}=\theta_{n-1}+K_{n} e_{n} x_{n}
$$

with

$$
\begin{aligned}
K_{n} & =\frac{1}{\lambda} & & \left(K_{n-1}-s\left(v_{n} v_{n}^{T}\right)\right) \\
s & = & & \frac{1}{\lambda+x_{n}^{T} v_{n}} \\
v_{n} & = & & K_{n-1} x_{n}
\end{aligned}
$$

where $\lambda$ was the forgetting factor and ${ }^{T}$ was the symbol of the transpose.

The signal $y(n)$ is compared to the one built by the model $\hat{y}(n)$ in order to minimize the error between the two signals. The optimization adapted itself over time.

Finally, the switch was to position 2. The modeled signal $\hat{y}(n)$ is reversed and amplified so that the power of $x(n)$ was equal to the energy $\hat{y}(n)$. 


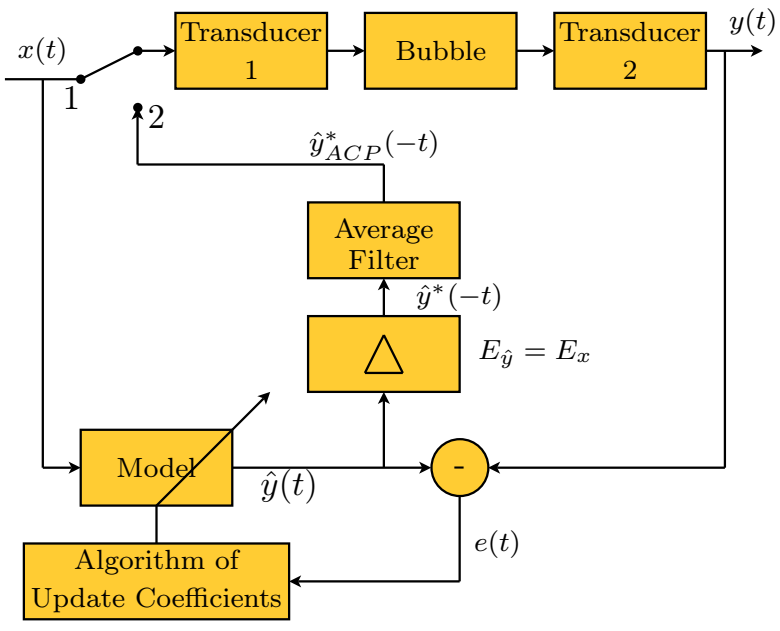

Fig. 3

Scheme of the bubble SYSTem ASSOciated With the IDENTIFICATION SYSTEM

\section{C.3 Average Filter}

As the signal to noise ratio (SNR) of the filtered signal $y(t)$ was low (due to the incoherent variations between each echo), we proposed to increase the SNR by building the signal $y_{A C P}(t)$ from a linear combination of the filtered signal $y(t)$, i.e. principal component analysis (PCA) 9] such as :

$$
\mathbf{y}_{\mathbf{A C P}}=\mathbf{A}^{\prime} \mathbf{y}^{*},
$$

where $\mathbf{y}_{\mathbf{A C P}}$ was the principal component score, A was composed of the eigenvectors of the correlation matrix and $\mathbf{y}^{*}$ was the matrix composed of the vectors of the standardized $\mathbf{y}$. The new signal $y_{A C P}(t)$ from which the power was evaluated in the optimization process corresponded to the first column in the matrix $\mathbf{y}_{\mathbf{A C P}}$.

\section{Results \& Discussions}

\section{A. Simulation Without Noise}

The bubble was initially excited by a sinusoid modulated by a Gaussian amplitude of $137 \mathrm{kPa}$ (figure 4). The

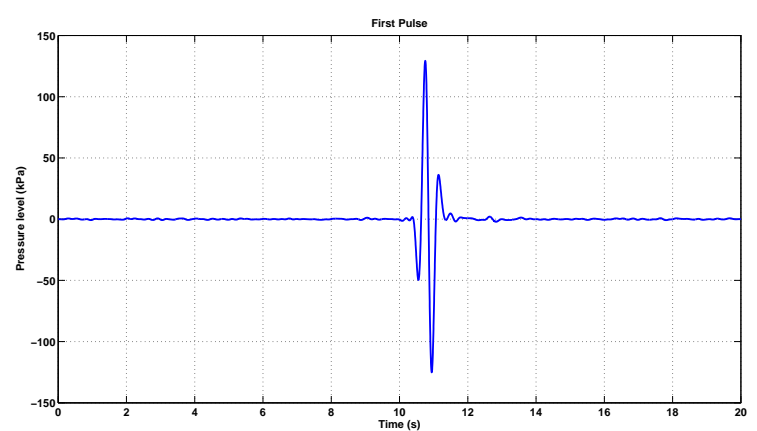

Fig. 4

INITIAL PULSE With A PRESSURE LEVEL OF $137 \mathrm{KPA}$ backscattered signal was modeled by the RLS algorithm (figure 5).

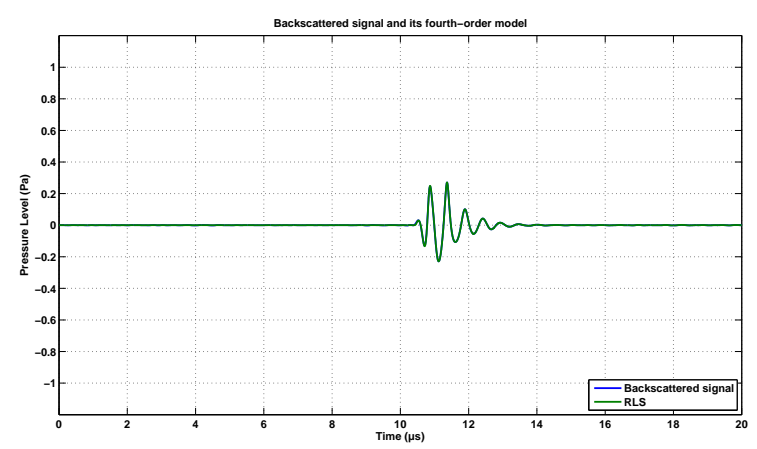

Fig. 5

Signal BACKSCATtered By THE MICROBUbBLE

The model of the signal was reversed and amplified so that its power was equal to that of the initial sinusoidal excitation. The signal of figure 6 was the best signal.

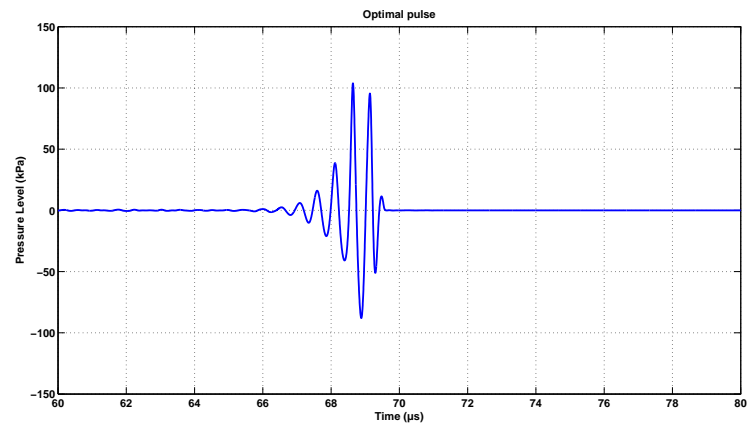

Fig. 6

Optimal PUlSe With the SAME POWER than the Initial PUlSE

When the bubble is excited by the optimal pulse (figure 17), the backscattered power achieves a gain of $17.68 \mathrm{~dB}$ compared to the backscattered power without optimisation process.

The identification of the bubble system as a filter allowed us to optimize the power response of the microbubble. This optimization did not require a priori knowledge of the system. It optimized the system bubble consituted by a cloud of microbubbles and the transducers.

\section{B. Simulation With Noise}

The initial pulse is a sinus wave with a white noise which had the same power than the sinusoidal signal modulated by a Gaussian presented in figure 4, The signal to noise ratio (SNR) was $4.74 \mathrm{~dB}$. The order of the RLS filter was compared to find the optimal order in an empirical way. The experiment is repeated for several different orders. The optimal order was 4 (figure 8).

Moreover, we tested two possible configurations. The first was the standard time-reversal technique. This opti- 


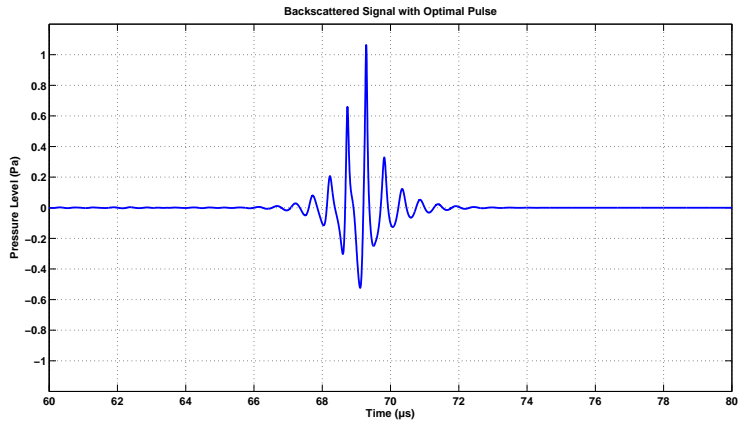

Fig. 7

SignAl BACKSCATtered FROM THE OPtimal PULSE

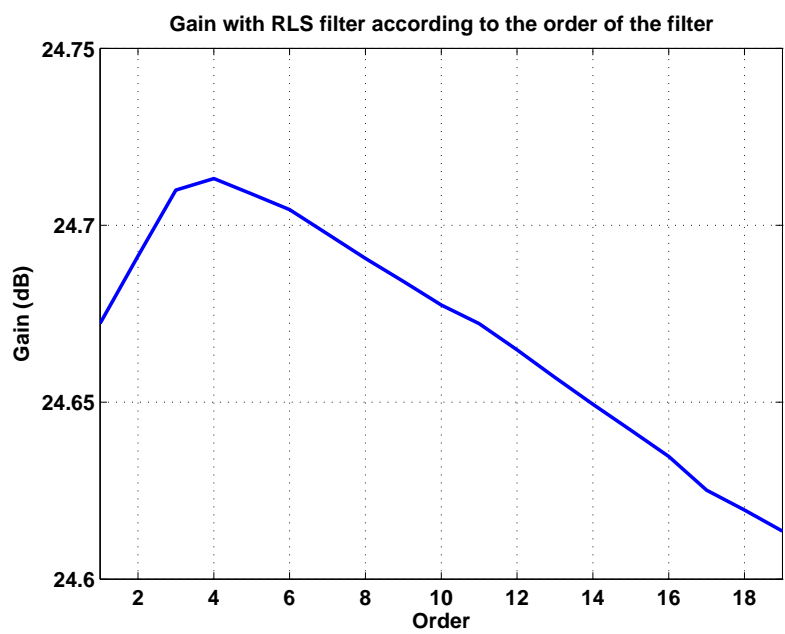

Fig. 8

Gain of the optimization ACCORding to The ORder of the RLS FILTER

mization allowed us to reach a gain of $10.85 \mathrm{~dB}$ compared to the signal backscattered from the initial pulse. The second was an identification system using modeling filter with the RLS filter. This second optimization allows us to obtain a gain of $24.72 \mathrm{~dB}$.

This last simulation confirmed that the identification system optimized the power backscattered by the bubble. Moreover, this simulation showed that the gain was increased by $13.87 \mathrm{~dB}$ using a filter such as the RLS filter. The RLS filter was able to extract the essential information to identify the bubble system.

\section{Experiment}

The experiment consisted in transmitting, through the cloud of microbubbles, a sinusoid modulated by a Gaussian with a pressure of $137 \mathrm{kPa}$ with the same characteristics as the stimulus presented in figure 4. Both identifications were compared: the first used the data from the backscattered signal while the second used the signal modeled by the filter RLS. The identification system was performed several times, which corresponds to iterations. The backscattered signal at iteration $n$ was again used as initial pulse for the next iteration $n+1$.

The gain of the optimization can reach $4.6 \mathrm{~dB}$ (figure 9) compared to the backscattered power of sinus pulse in the case of the matched filter. If the identification by RLS filter was used, the gain could reach $6.3 \mathrm{~dB}$.

As in our simulations, the RLS filter can increase the gain of the optimization. The experimental results are not exactly identical because of the non-stationarity of microbubbles. To cancel the fluctuation due to their motion, we have repeated the experiment. Since a high number of repetition and a high value of iterations number could destroy the microbubbles and thus induce a backscattered power reduction, a trade-off must be found to avoid the destruction of the microbubbles.

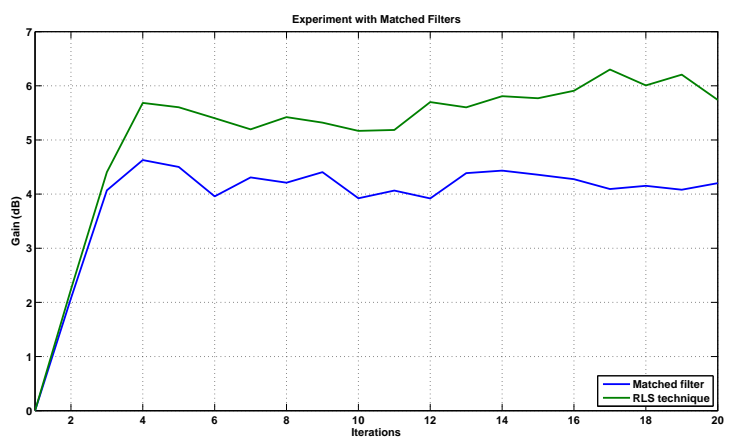

Fig. 9

EXPERIMENT REALIZED WITH AN INITIAL SinUs PULSE OF PRESSURE LEVEL OF 137 KPA

\section{CONClusion}

The use of matched filter (time reversal technique) seems a good choice to optimize the CTR. Among the two proposed techniques, we suggest to use the RLS time reversal technique.

We can carry on modeling the signal by nonlinear filters like Volterra filter and implementing the RLS filter in an echograph with an programmable generator.

\section{ACKNOWLEGDEMENT}

The authors would like to acknowledge Ayache Bouakaz for the ultrasound contrast agents.

This work was supported by Agence Nationale de la Recherche (Project ANR 07, Tecsan 015, MONitoring response to THERapy with a novel ultrasound technology).

\section{REFERENCES}

[1] S. Ménigot, A. Novell, A. Bouakaz, and J.-M. Girault, "Improvement of the power response in contrast imaging with transmit frequency optimization," in Proceeding IEEE Ultrasonic Symposium, 2009, pp. 1247-1250.

[2] B. Widrow and S. Stearns, Adaptive Signal Processing. Englewood Cliffs, New Jersey, USA: Prentice Hall, 1985.

[3] C. Greis, "Technology overview: Sonovue (bracco, milan)," Eur Radiol Suppl, vol. 14, no. 8, pp. 11-15, October 2004. 
[4] H. J. Vos, F. Guidi, E. Boni, and P. Tortoli, "Acoustical investigation of freely moving single microbubbles," in Proc IEEE Ultrason Symp, vol. 2, 18-21 Sept. 2005, pp. 755-758.

[5] K. Chetty, C. Sennoga, J. Hainal, R. Eckersley, and E. Stride, "P1f-4 high speed optical observations and simulation results of lipid based microbubbles at low insonation pressures," in Proc IEEE Ultrason Symp, 2-6 Oct. 2006, pp. 1354-1357.

[6] H. Vos, F. Guidi, E. Boni, and P. Tortoli, "Method for microbubble characterization using primary radiation force," IEEE Trans Ultrason Ferroelectr Freq Control, vol. 54, no. 7, pp. 1333-1345, July 2007.

[7] L. Hoff, Acoustic Characterization of Contrast Agents for Medical Ultrasound Imaging. Boston: Kluwer Academic, 2001.

[8] F. Michaut and M. Bellanger, Filtrage adaptatif : théorie et algorithmes. Paris, France: Hermes Science, 2005.

[9] I. T. Jolliffe, Principal Component Analysis, 2nd ed. New York, NY, USA: Springer, October 2002. 